MATHEMATICS OF COMPUTATION

Volume 75, Number 253, Pages 327-343

S 0025-5718(05)01773-4

Article electronically published on August 31, 2005

\title{
EXTENSION OF A CLASS OF PERIODIZING VARIABLE TRANSFORMATIONS FOR NUMERICAL INTEGRATION
}

\author{
AVRAM SIDI
}

\begin{abstract}
Class $\mathcal{S}_{m}$ variable transformations with integer $m$, for numerical computation of finite-range integrals, were introduced and studied by the author in the paper [A. Sidi, A new variable transformation for numerical integration, Numerical Integration IV, 1993 (H. Brass and G. Hämmerlin, eds.), pp. 359-373.] A representative of this class is the $\sin ^{m}$-transformation that has been used with lattice rules for multidimensional integration. These transformations "periodize" the integrand functions in a way that enables the trapezoidal rule to achieve very high accuracy, especially with even $m$. In the present work, we extend these transformations to arbitrary values of $m$, and give a detailed analysis of the resulting transformed trapezoidal rule approximations. We show that, with suitable $m$, they can be very useful in different situations. We prove, for example, that if the integrand function is smooth on the interval of integration and vanishes at the endpoints, then results of especially high accuracy are obtained by taking $2 m$ to be an odd integer. Such a situation can be realized in general by subtracting from the integrand the linear interpolant at the endpoints of the interval of integration. We also illustrate some of the results with numerical examples via the extended $\sin ^{m}$-transformation.
\end{abstract}

\section{INTRODUCTION}

Consider the problem of evaluating finite-range integrals of the form

$$
I[f]=\int_{0}^{1} f(x) d x
$$

where $f \in C^{\infty}(0,1)$ but is not necessarily continuous or differentiable at $x=0$ and $x=1$. $f(x)$ may even behave singularly at the endpoints, with different types of singularities. One very effective way of computing $I[f]$ is by first transforming it with a suitable variable transformation and next applying the trapezoidal rule to the resulting transformed integral. Thus, if we make the substitution $x=\psi(t)$, where $\psi(t)$ is an increasing differentiable function on $[0,1]$, such that $\psi(0)=0$ and $\psi(1)=1$, then the transformed integral is

$$
I[f]=\int_{0}^{1} \widehat{f}(t) d t ; \quad \widehat{f}(t)=f(\psi(t)) \psi^{\prime}(t),
$$

Received by the editor December 2, 2003 and, in revised form, August 16, 2004.

2000 Mathematics Subject Classification. Primary 30E15, 40A25, 41A60, 65B15, 65D30, $65 \mathrm{D} 32$.

Key words and phrases. Numerical integration, variable transformations, $\sin ^{m}$-transformation, Euler-Maclaurin expansions, asymptotic expansions, trapezoidal rule.

(C)2005 American Mathematical Society Reverts to public domain 28 years from publication 
and the trapezoidal rule approximation to $I[f]$ is

$$
\widehat{Q}_{n}[f]=h\left[\frac{1}{2} \widehat{f}(0)+\sum_{i=1}^{n-1} \widehat{f}(i h)+\frac{1}{2} \widehat{f}(1)\right], \quad h=\frac{1}{n} .
$$

[Normally, we also demand that $\psi(1-t)=1-\psi(t)$, which forces on $\psi^{\prime}(t)$ the symmetry property $\psi^{\prime}(1-t)=\psi^{\prime}(t)$.] If, in addition, $\psi(t)$ is chosen such that $\psi^{(i)}(0)=\psi^{(i)}(1)=0, i=1,2, \ldots, p$, for some sufficiently large $p$, then $\widehat{Q}_{n}[f]$, even for moderate $n$, approximate $I[f]$ with surprisingly high accuracy. In such a case, we may have $\widehat{f}(0)=\widehat{f}(1)=0$, and $\widehat{Q}_{n}[f]$ becomes

$$
\widehat{Q}_{n}[f]=h \sum_{i=1}^{n-1} \widehat{f}(i h) .
$$

Variable transformations in numerical integration have been of considerable interest lately. In the context of one-dimensional integration, they are used as a means to improve the performance of the trapezoidal rule. In the context of multidimensional integration, they are used to "periodize" the integrand in all variables so as to improve the accuracy of lattice rules. (Lattice rules are extensions of the trapezoidal rule to many dimensions.)

There is a whole collection of variable transformations in the literature of numerical integration. We mention here the polynomial transformation of Korobov [9], the tanh-transformation of Sag and Szekeres [15, the IMT-transformation of Iri, Moriguti, and Takasawa [7, the double exponential formula of Mori [12, the class $\mathcal{S}_{m}$ transformations ( $m$ is a positive integer) of Sidi [17, and the polynomial transformation of Laurie [10].

In this paper, we concentrate on the class $\mathcal{S}_{m}$ transformations of the author, which have some very interesting and useful properties when coupled with the trapezoidal rule. A trigonometric representative of these, namely, the $\sin ^{m}$-transformation that was also proposed and studied in [17, has been used successfully in conjunction with lattice rules in multiple integration; see Sloan and Joe 223, Hill and Robinson [6], and Robinson and Hill [14]. The $\sin ^{m}$-transformation has also been used in the computation of multidimensional integrals in conjunction with extrapolation methods by Verlinden, Potts, and Lyness [24].

Another trigonometric transformation similar to the $\sin ^{m}$-transformation was recently given by Elliott [4, and this transformation, too, is in the class $\mathcal{S}_{m}$ with even $m$. The polynomial transformation of Laurie was designed to have some of the useful properties of class $\mathcal{S}_{m}$ transformations, but is not in $\mathcal{S}_{m}$.

In the next section, we extend the definition of the class $\mathcal{S}_{m}$ to noninteger values of $m$. Following that, in Section 3, we extend the definition of the $\sin ^{m}$ transformation accordingly. We show how to compute this extended transformation economically. In Section 4, we analyze the properties of the extended class $\mathcal{S}_{m}$ transformations in conjunction with the trapezoidal rule, and we show how to select $m$ optimally in any given situation. In Section 5, we illustrate the theoretical results of Section 4 with numerical examples via the extended $\sin ^{m}$-transformation.

Before proceeding further, we would like to introduce the concept of the quality of the numerical quadrature rule $\widehat{Q}_{n}[f]$ computed in conjunction with a variable transformation $\psi(t)$ that behaves like a power of $t$ as $t \rightarrow 0+$. 
Definition 1.1. Let $f(x), I[f], \psi(t), \widehat{f}(t)$, and $\widehat{Q}_{n}[f]$ be as in the first paragraph of this section. Let the variable transformation $\psi(t)$ have the property that

$$
\psi(1-t)=1-\psi(t) .
$$

Also assume that, for some $q \geq 1, \psi(t) \sim \alpha t^{q}$ as $t \rightarrow 0+\left[\right.$ hence $\psi(t) \sim 1-\alpha(1-t)^{q}$ as $t \rightarrow 1-$ as well]. If $\widehat{Q}_{n}[f]-I[f]=O\left(h^{\sigma}\right)$ as $h \rightarrow 0$, then the quality of $\widehat{Q}_{n}[f]$ is the ratio $\sigma / q$.

To motivate this concept, let us go back to the transformed quadrature rule $\widehat{Q}_{n}[f]$ in (1.4). The fact that $\psi(t) \sim \alpha t^{q}$ as $t \rightarrow 0$ implies that, when $q$ is large, the abscissas of the rule $\widehat{Q}_{n}[f]$, namely, $x_{i} \equiv \psi(i h)=\psi(i / n)$ in the original variable of integration $x$, are clustered in two very small regions, one to the right of $x=0$ and the other to the left of $x=1$, many of them being very close to 0 and to 1 . The amount of this clustering is determined by the size of $q$; the larger $q$, the larger the density of the $x_{i}$ near $x=0$ and $x=1$. As the clustering gets larger, the numerical computation of the rule $\widehat{Q}_{n}[f]$ in finite-precision arithmetic may become problematic due to possible underflows and overflows in case $f(x)$ has endpoint singularities. From this, we conclude that too much clustering is not desirable. Thus, for a given $q$ (that is, for a given amount of clustering), we would like to get as high an accuracy as possible out of $\widehat{Q}_{n}[f]$. A good measure of this would thus be the ratio $\sigma / q$, the quality of $\widehat{Q}_{n}[f]$; the higher the quality, the better the rule. Therefore, this ratio also enables us to compare different variable transformations in a convenient way.

One of the results of Section 4 concerns the case when $f \in C^{\infty}[a, b]$ and $f(x)$ vanishes at $x=0$ and $x=1$. In such a case, exceptionally high accuracy is obtained from $\widehat{Q}_{n}[f]$ via a transformation from $\mathcal{S}_{m}$ with $2 m$ an odd integer. Indeed, we show that the quality of $\widehat{Q}_{n}[f]$ for such integrals is at least 3 when $2 m$ is an odd integer, whereas it is 2 for all other values of $m$, integer or otherwise. If also $f^{\prime \prime}(x)$ vanishes at the endpoints, the quality of this $\widehat{Q}_{n}[f]$ becomes at least 4 . The quality increases as further derivatives of consecutive orders of $f(x)$ vanish at the endpoints. This fact is now used in approximating $I[f]$ as follows: Subtract from $f(x)$ the linear function $p(x)$ that interpolates it at $x=0$ and $x=1$, and approximate $I[f]$ via $\widehat{Q}_{n}[f-p]+I[p]$, with $2 m$ an odd integer, $I[p]=\int_{0}^{1} p(x) d x$ being known exactly. Clearly, the new integrand $f(x)-p(x)$ vanishes at $x=0$ and $x=1$, and the transformed rule $\widehat{Q}_{n}[f-p]+I[f]$ has quality at least 3 . [Of course, if other suitable functions $p(x)$, whose integrals can be computed exactly, are known, these can be used in a similar fashion.] This shows that preprocessing $f(x)$ appropriately can increase the quality of transformed quadrature rules substantially.

1.1. Technical preliminaries. Euler-Maclaurin expansions concerning the trapezoidal rule approximations of finite-range integrals $\int_{a}^{b} u(x) d x$ are the main analytical tool we use in our study. For the sake of easy reference, here we reproduce the relevant Euler-Maclaurin expansion due to the author (see Sidi [20, Corollary 2.2]) as Theorem 1.2. This theorem is a special case of another very general theorem from [20, is expressed in terms of the asymptotic expansions of $u(x)$ as $x \rightarrow a+$ and $x \rightarrow b-$, and is easy to write down and use. 
Theorem 1.2. Let $u \in C^{\infty}(a, b)$, and assume that $u(x)$ has the asymptotic expansions

$$
\begin{aligned}
& u(x) \sim \sum_{s=0}^{\infty} c_{s}(x-a)^{\gamma_{s}} \quad \text { as } x \rightarrow a+, \\
& u(x) \sim \sum_{s=0}^{\infty} d_{s}(b-x)^{\delta_{s}} \quad \text { as } x \rightarrow b-,
\end{aligned}
$$

where the $\gamma_{s}$ and $\delta_{s}$ are distinct complex numbers that satisfy

$$
\begin{aligned}
& -1<\Re \gamma_{0} \leq \Re \gamma_{1} \leq \Re \gamma_{2} \leq \cdots, \quad \lim _{s \rightarrow \infty} \Re \gamma_{s}=+\infty, \\
& -1<\Re \delta_{0} \leq \Re \delta_{1} \leq \Re \delta_{2} \leq \cdots, \quad \lim _{s \rightarrow \infty} \Re \delta_{s}=+\infty .
\end{aligned}
$$

Assume furthermore that, for each positive integer $k, u^{(k)}(x)$ has asymptotic expansions as $x \rightarrow a+$ and $x \rightarrow b-$ that are obtained by differentiating those of $u(x)$ term by term $k$ times. Also let $h=(b-a) / n$ for $n=1,2, \ldots$. Then

$$
\begin{aligned}
h \sum_{i=1}^{n-1} u(a+i h) \sim \int_{a}^{b} u(x) d x & +\sum_{\substack{s=0 \\
\gamma_{s} \notin\{2,4,6, \ldots\}}}^{\infty} c_{s} \zeta\left(-\gamma_{s}\right) h^{\gamma_{s}+1} \\
& +\sum_{\substack{s=0 \\
\gamma_{s} \notin\{2,4,6, \ldots\}}}^{\infty} d_{s} \zeta\left(-\delta_{s}\right) h^{\delta_{s}+1} \text { as } h \rightarrow 0,
\end{aligned}
$$

where $\zeta(z)$ is the Riemann Zeta function.

It is clear from Theorem 1.2 that even powers of $(x-a)$ and $(b-x)$, if present in the asymptotic expansions of $u(x)$ as $x \rightarrow a+$ and $x \rightarrow b-$, do not contribute to the asymptotic expansion of $h \sum_{i=1}^{n-1} u(a+i h)$ as $h \rightarrow 0$.

In addition, if $\gamma_{p}$ is the first of the $\gamma_{s}$ that is different from $2,4,6, \ldots$, and if $\delta_{q}$ is the first of the $\delta_{s}$ that is different from $2,4,6, \ldots$, then

$$
h \sum_{i=1}^{n-1} u(a+i h)-\int_{a}^{b} u(x) d x=O\left(h^{\sigma+1}\right) \quad \text { as } h \rightarrow 0, \quad \sigma=\min \left\{\Re \gamma_{p}, \Re \delta_{q}\right\} .
$$

This is a useful observation that we will recall later.

Of course, when $u \in C^{\infty}[a, b]$, the expansion given in Theorem 1.2 reduces to the classical Euler-Maclaurin expansion for the trapezoidal rule, as it must. This can be seen by observing that now the $\gamma_{s}$ and $\delta_{s}$ are nonnegative integers, so that $c_{s}=u^{\left(\gamma_{s}\right)}(a) / \gamma_{s}$ ! and $d_{s}=(-1)^{\delta_{s}} u^{\left(\delta_{s}\right)}(b) / \delta_{s}$ !, and by invoking the known facts that $\zeta(0)=-1 / 2$ and $\zeta(-2 j)=0$ and $\zeta(1-2 j)=-B_{2 j} /(2 j)$ for $j=1,2, \ldots$, where $B_{s}$ is the $s$ th Bernoulli number. Thus,

$$
\begin{aligned}
h \sum_{i=1}^{n-1} u(a+i h) \sim \int_{a}^{b} u(x) d x & -\frac{h}{2}[u(a)+u(b)] \\
& +\sum_{k=1}^{\infty} \frac{B_{2 k}}{(2 k) !}\left[u^{(2 k-1)}(b)-u^{(2 k-1)}(a)\right] h^{2 k} \quad \text { as } h \rightarrow 0 .
\end{aligned}
$$

For the treatment of the classical Euler-Maclaurin expansion, see, for example, Davis and Rabinowitz [3], Ralston and Rabinowitz [13], and Atkinson [2]. See also the brief review in Sidi [19, Appendix D]. 


\section{Extended Class $\mathcal{S}_{m}$ TRANSFORMATIONS}

Definition 2.1. A function $\psi(t)$ is in the extended class $\mathcal{S}_{m}, m$ arbitrary, if it has the following properties:

1. $\psi \in C[0,1]$ and $\psi \in C^{\infty}(0,1) ; \psi(0)=0, \psi(1)=1$, and $\psi^{\prime}(t)>0$ on $(0,1)$.

2. $\psi^{\prime}(t)$ is symmetric with respect to $t=1 / 2$; that is, $\psi^{\prime}(1-t)=\psi^{\prime}(t)$. Consequently, $\psi(1-t)=1-\psi(t)$.

3. $\psi^{\prime}(t)$ has the following asymptotic expansions as $t \rightarrow 0+$ and $t \rightarrow 1-$ :

$$
\begin{aligned}
& \psi^{\prime}(t) \sim \sum_{i=0}^{\infty} \epsilon_{i} t^{m+2 i} \quad \text { as } t \rightarrow 0+ \\
& \psi^{\prime}(t) \sim \sum_{i=0}^{\infty} \epsilon_{i}(1-t)^{m+2 i} \quad \text { as } t \rightarrow 1-
\end{aligned}
$$

the $\epsilon_{i}$ being the same in both expansions, and $\epsilon_{0}>0$. Consequently,

$$
\begin{aligned}
& \psi(t) \sim \sum_{i=0}^{\infty} \epsilon_{i} \frac{t^{m+2 i+1}}{m+2 i+1} \quad \text { as } t \rightarrow 0+ \\
& \psi(t) \sim 1-\sum_{i=0}^{\infty} \epsilon_{i} \frac{(1-t)^{m+2 i+1}}{m+2 i+1} \quad \text { as } t \rightarrow 1-.
\end{aligned}
$$

4. Furthermore, for each positive integer $k, \psi^{(k)}(t)$ has asymptotic expansions as $t \rightarrow 0+$ and $t \rightarrow 1$ - that are obtained by differentiating those of $\psi(t)$ term by term $k$ times.

The difference between Definition 2.1 and the definition of the class $\mathcal{S}_{m}$ in 17 is that $m$ is a positive integer in the latter, hence $\psi \in C^{\infty}[0,1]$. In Definition 2.1. $\psi(t)$ is not infinitely differentiable at $t=0$ and $t=1$ when $m$ is not a positive integer. The fact that we are now allowing $m$ to assume arbitrary values has a very beneficial effect, as mentioned in Section 1 and as we will see in the next sections.

As was mentioned in 17, the fact that $\psi^{\prime}(t)$ has the asymptotic expansions given in (2.1) - with consecutive powers of $t$ and $(1-t)$ there increasing by 2 instead of by 1 - is the most important aspect of the extended class $\mathcal{S}_{m}$.

The following result shows that the family of the extended classes $\mathcal{S}_{m}$ is closed with respect to composition. It is an immediate extension of Lemma 2.1 in [17.

Lemma 2.2. Let $\psi_{i} \in \mathcal{S}_{m_{i}}, i=1, \ldots, r$, and define $\Psi(t)=\psi_{1}\left(\psi_{2}\left(\cdots\left(\psi_{r}(t)\right) \cdots\right)\right)$. Then $\Psi \in \mathcal{S}_{M}$ with $M=\prod_{i=1}^{r}\left(m_{i}+1\right)-1$.

The following are special cases of Lemma 2.2.

(i) In case $m_{i}$ are all integers, $M$ is an integer, too; $M$ is an even integer if and only if $m_{i}$ are all even integers.

(ii) In case $r=2$ [so that $\Psi(t)=\psi_{1}\left(\psi_{2}(t)\right) \in \mathcal{S}_{M}$ with $M=$ $\left.\left(m_{1}+1\right)\left(m_{2}+1\right)-1\right]$, and $m_{1}, m_{2} \in\{k / 2: k=1,2, \ldots\}, 2 M$ is an odd integer if and only if $m_{1}$ is an even integer and $2 m_{2}$ is an odd integer, or vice versa.

This lemma shows that transformations different from the $\sin ^{m}$-transformation can be obtained, for example, by composing two or more of the latter with various values of $m$. 
Before proceeding further, we recall that, if the integrand $f(x)$ in the integral $\int_{0}^{1} f(x) d x$ is sufficiently smooth in $[0,1]$, and if we let $x=\psi(t)$ with $\psi \in \mathcal{S}_{m}$, then excellent approximations are obtained by applying the trapezoidal rule to the transformed integral $\int_{0}^{1} f(\psi(t)) \psi^{\prime}(t) d t$ when $m$ is an even integer, and the error in this approximation is at worst $O\left(n^{-2 m-2}\right)$ as $n \rightarrow \infty$, where $n-1$ is the number of abscissas in the approximation, as shown in [17]. Now, by (2.2), $\psi \in \mathcal{S}_{m}$ behaves asymptotically (in a polynomial fashion) as in

$$
\psi(t) \sim \alpha t^{m+1} \quad \text { as } t \rightarrow 0, \quad \psi(t) \sim 1-\alpha(1-t)^{m+1} \quad \text { as } t \rightarrow 1 .
$$

If, instead of class $\mathcal{S}_{m}$ transformations, we use the Korobov transformation that also behaves asymptotically in the same way, the error in the resulting approximations to $\int_{0}^{1} f(x) d x$ is at worst $O\left(n^{-m-2}\right)$ as $n \rightarrow \infty$, when $m$ is an even integer. This shows that the transformed rules $\widehat{Q}_{n}[f]$ obtained using class $\mathcal{S}_{m}$ transformations have more useful approximation properties. In the sense of Definition 1.1, they have higher quality.

\section{THE EXTENDED $\sin ^{m}$-TRANSFORMATION}

The extended $\sin ^{m}$-transformation, just as the original $\sin ^{m}$-transformation, is defined via

$$
\psi_{m}(t)=\frac{\Theta_{m}(t)}{\Theta_{m}(1)} ; \quad \Theta_{m}(t)=\int_{0}^{t}(\sin \pi u)^{m} d u .
$$

From the equality

$$
\Theta_{m}(t)=\frac{m-1}{m} \Theta_{m-2}(t)-\frac{1}{\pi m}(\sin \pi t)^{m-1} \cos \pi t,
$$

which can be obtained by integration by parts, we have the recursion relation

$$
\psi_{m}(t)=\psi_{m-2}(t)-\frac{\Gamma\left(\frac{m}{2}\right)}{2 \sqrt{\pi} \Gamma\left(\frac{m+1}{2}\right)}(\sin \pi t)^{m-1} \cos \pi t .
$$

This recursion relation is valid for all $m$. Also, $\psi_{m}(t)$ is related to $\psi_{m-2}(t)$ but not to $\psi_{m-1}(t)$. Thus, the sequences $\left\{\psi_{2 k+\omega}(t)\right\}_{k=0}^{\infty}$ and $\left\{\psi_{2 k+1+\omega}(t)\right\}_{k=0}^{\infty}$, with $0 \leq \omega<1$, can be computed independently of each other, with $\psi_{\omega}(t)$ and $\psi_{1+\omega}(t)$, respectively, as the initial conditions. The recursion relation in (3.2) is, of course, stable in the forward direction.

When $m$ is a positive integer, thus $\omega=0, \psi_{m}(t)$ can be expressed in terms of elementary functions, and (3.2), with the initial conditions

$$
\psi_{0}(t)=t \quad \text { and } \quad \psi_{1}(t)=\frac{1}{2}(1-\cos \pi t)
$$

can be used to compute $\psi_{m}(t)$. When $m$ is not an integer, however, $\psi_{m}(t)$ does not seem to have a representation in terms of elementary functions, and we need a different approach to its computation.

3.1. Computation for $m$ not an integer. We now turn to the actual computation of this transformation when $m$ is not an integer. We do this by computing $\Theta_{m}(t)$ in 3.1). By the fact that $\Theta_{m}^{\prime}(t)=(\sin \pi t)^{m}$ is symmetric with respect to $t=1 / 2$, we have that $\Theta_{m}(t)=\Theta_{m}(1)-\Theta_{m}(1-t)$ for $t \in[1 / 2,1]$ and thus 
$\Theta_{m}(1)=2 \Theta_{m}(1 / 2)$ as well. Thus, it is enough to know $\Theta_{m}(t)$ for $t \in[0,1 / 2]$. Consequently,

$$
\psi_{m}(t)=\frac{\Theta_{m}(t)}{2 \Theta_{m}(1 / 2)} \text { for } t \in[0,1 / 2], \quad \psi_{m}(t)=1-\psi_{m}(1-t) \text { for } t \in[1 / 2,1] .
$$

Therefore, in the remainder of this section, we consider the computation of $\Theta_{m}(t)$ only for $t \in[0,1 / 2]$.

3.1.1. Via numerical integration. The first way is by computing the integral representation of $\Theta(t)$ numerically. Now,

$$
\Theta(t)=\int_{0}^{t} u^{m} R(u) d u, \quad R(u)=\left(\frac{\sin \pi u}{u}\right)^{m} .
$$

Because $R(u)$ is infinitely smooth on $[0,1 / 2]$, this integral can be computed with high accuracy using Gauss-Jacobi quadrature with weight function $u^{m}$ when $m$ is real. Of course, this necessitates the availability of the abscissas and weights for these quadrature formulas for each value of $m$. These abscissas and weights can be computed with the aid of Gautschi's ORTHPOL package [5].

3.1.2. Via hypergeometric series. It turns out that $\Theta_{m}(t)$ can be expressed in terms of the Gauss hypergeometric function $F(a, b ; c ; z)={ }_{2} F_{1}(a, b ; c ; z)$ in several ways. For the definition and properties of $F(a, b ; c ; z)$, see, for example, Abramowitz and Stegun [1, Chapter 15].

By [1, p. 556, formula 15.1.1],

$$
F(a, b ; c ; z)=\sum_{k=0}^{\infty} \frac{(a)_{k}(b)_{k}}{(c)_{k}} \frac{z^{k}}{k !}
$$

where $(x)_{0}=1$ and $(x)_{k}=x(x+1) \cdots(x+k-1)$ for $k=1,2, \ldots$. Now, the $k$ th term of this series tends to zero practically like $z^{k}$ when $|z|<1$. This suggests that some of the (hypergeometric) series representations of $\Theta_{m}(t)$ can also be used to actually compute $\Theta_{m}(t)$ for noninteger $m$ efficiently. One advantage of this approach is that the terms $w_{k}=\frac{(a)_{k}(b)_{k}}{(c)_{k}} \frac{z^{k}}{k !}$ can be computed at a small cost by the recursion relation $w_{k+1}=\frac{(a+k)(b+k)}{(c+k)} \frac{z}{k+1} w_{k}$.

In the following, we give two such representations, which are of interest in themselves:

(i) Making the substitution $\xi=\sin (\pi u / 2)$ in (3.1), which is legitimate because $\sin (\pi u / 2)$ is an increasing function for $u \in[0, t]$ when $t \in[0,1]$, we obtain

$$
\Theta_{m}(t)=\frac{2^{m+1}}{\pi} \int_{0}^{S} \xi^{m}\left(\sqrt{1-\xi^{2}}\right)^{m-1} d \xi, \quad S=\sin \frac{\pi t}{2} .
$$

Expanding the integrand about $\xi=0$ and integrating the resulting (absolutely and uniformly convergent) power series term by term, we obtain

$$
\Theta_{m}(t)=\frac{(2 S)^{m+1}}{\pi} \sum_{k=0}^{\infty} \frac{\left(\frac{1-m}{2}\right)_{k}}{k !} \frac{S^{2 k}}{m+2 k+1}, \quad S=\sin \frac{\pi t}{2},
$$

which can be expressed in terms of the hypergeometric function as in

$$
\Theta_{m}(t)=\frac{(2 S)^{m+1}}{\pi(m+1)} F\left(\frac{1}{2}-\frac{1}{2} m, \frac{1}{2} m+\frac{1}{2} ; \frac{1}{2} m+\frac{3}{2} ; S^{2}\right), \quad S=\sin \frac{\pi t}{2} .
$$


For $k \geq\lfloor(m+1) / 2\rfloor$, the terms in this expansion are of the same sign, and tend to zero as $k \rightarrow \infty$ essentially like $k^{-(m+3) / 2} S^{2 k}$, and hence, by the fact that $0 \leq S \leq \sin (\pi / 4)=1 / \sqrt{2}$, at worst like $k^{-(m+3) / 2} 2^{-k}$. Thus, the expansion in (3.4) converges quickly and can be used for the actual computation of $\Theta_{m}(t)$. Furthermore, we can also use a nonlinear sequence transformation, such as that of Shanks [16] (or the equivalent $\epsilon$-algorithm of Wynn [25]) or of Levin [11, to accelerate the convergence of this expansion. Both transformations are treated in detail in the recent book by Sidi 19 .

(ii) Invoking in (3.5) one of the so-called linear transformation formulas (see 1,

p. 559, formulas 15.3 .4 and 15.3 .5$]$ ), and using the fact $\frac{S^{2}}{S^{2}-1}=-\tan ^{2} \frac{\pi t}{2}$ and the relations

$$
\sin x=\frac{\tan x}{\sqrt{1+\tan ^{2} x}} \quad \text { and } \quad \cos x=\frac{1}{\sqrt{1+\tan ^{2} x}}, \quad 0 \leq x \leq \frac{\pi}{2},
$$

we obtain

$\Theta_{m}(t)=\frac{2 T}{\pi(m+1)}\left(\frac{2 T}{1+T^{2}}\right)^{m} F\left(1, \frac{1}{2}-\frac{1}{2} m ; \frac{1}{2} m+\frac{3}{2} ;-T^{2}\right), \quad T=\tan \frac{\pi t}{2}$,

which has the expansion

$$
\Theta_{m}(t)=\frac{2 T}{\pi(m+1)}\left(\frac{2 T}{1+T^{2}}\right)^{m} \sum_{k=0}^{\infty} \frac{\left(\frac{1}{2}-\frac{1}{2} m\right)_{k}}{\left(\frac{1}{2} m+\frac{3}{2}\right)_{k}}\left(-T^{2}\right)^{k}, \quad T=\tan \frac{\pi t}{2} .
$$

Since $T^{2} \leq 1$ for $0 \leq t \leq 1 / 2$, the terms of this series tend to zero like $k^{-m-1} T^{2 k}$ as $k \rightarrow \infty$. Consequently, this expansion converges very slowly for $t$ close to $1 / 2$ because $T^{2} \rightarrow 1$ as $t \rightarrow 1 / 2$. However, it is an essentially alternating series because, for $k \geq\lfloor(m+1) / 2\rfloor$, its terms alternate in sign. This being the case, the series in (3.7) turns out to be ideal for the actual computation of $\Theta_{m}(t)$, because we can apply to it the Shanks or the Levin transformation and obtain its sum to machine precision using a very small number of its terms and in an absolutely stable fashion. Indeed, using the Levin transformation, $\Theta_{m}(t)$ can be computed with an accuracy of almost thirty-five digits by using only the first twenty-five terms of the expansion in (3.7).

\section{Analysis of the trapezoidal RUle WITH EXTENDED CLASS $\mathcal{S}_{m}$ TRANSFORMATIONS}

In this section and the next, we call the extended class $\mathcal{S}_{m}$ simply the class $\mathcal{S}_{m}$, and extended class $\mathcal{S}_{m}$ transformations simply class $\mathcal{S}_{m}$ transformations.

The following theorem is the main result of this section. Note that, for simplicity of notation, we set $[a, b]=[0,1]$.

Theorem 4.1. Let $f \in C^{\infty}(0,1)$, and assume that $f(x)$ has the asymptotic expansions

$$
f(x) \sim \sum_{s=0}^{\infty} c_{s} x^{\gamma_{s}} \quad \text { as } \quad x \rightarrow 0+; \quad f(x) \sim \sum_{s=0}^{\infty} d_{s}(1-x)^{\delta_{s}} \quad \text { as } \quad x \rightarrow 1-.
$$

Here $\gamma_{s}$ and $\delta_{s}$ are distinct complex numbers that satisfy

$$
\begin{array}{ll}
-1<\Re \gamma_{0} \leq \Re \gamma_{1} \leq \Re \gamma_{2} \leq \cdots, & \lim _{s \rightarrow \infty} \Re \gamma_{s}=+\infty, \\
-1<\Re \delta_{0} \leq \Re \delta_{1} \leq \Re \delta_{2} \leq \cdots, & \lim _{s \rightarrow \infty} \Re \delta_{s}=+\infty .
\end{array}
$$


Assume furthermore that, for each positive integer $k, f^{(k)}(x)$ has asymptotic expansions as $x \rightarrow 0+$ and $x \rightarrow 1$ - that are obtained by differentiating those of $f(x)$ term by term $k$ times. Let $I[f]=\int_{0}^{1} f(x) d x$, and let us now make the transformation of variable $x=\psi(t)$, where $\psi \in \mathcal{S}_{m}$, in $I[f]$. Finally, let us approximate $I[f]$ via the trapezoidal rule $\widehat{Q}_{n}[f]=\sum_{i=1}^{n-1} f(\psi(i h)) \psi^{\prime}(i h)$, where $h=1 / n, n=1,2, \ldots$. Then the following hold:

(i) In the worst case,

$$
\widehat{Q}_{n}[f]-I[f]=O\left(h^{(\omega+1)(m+1)}\right) \quad \text { as } h \rightarrow 0 ; \quad \omega=\min \left\{\Re \gamma_{0}, \Re \delta_{0}\right\} .
$$

(ii) Let us merge the sets $C=\left\{\gamma_{0}, \gamma_{1}, \ldots\right\}$ and $D=\left\{\delta_{0}, \delta_{1}, \ldots\right\}$ to obtain the set $B=\left\{\beta_{0}, \beta_{1}, \ldots\right\}$, such that (i) $\beta_{s}$ are distinct, (ii) $\Re \beta_{0} \leq \Re \beta_{1} \leq \cdots$, and (iii) $\alpha \in B$ if and only if $\alpha \in C$ or $\alpha \in D$. Then, if $\beta_{0}$ is real, and if $m=\left(q-\beta_{0}\right) /\left(1+\beta_{0}\right)$, where $q$ is an arbitrary positive even integer, then the preceding result is improved to read at worst

$$
\widehat{Q}_{n}[f]-I[f]=O\left(h^{\left(\beta_{1}+1\right)(m+1)}\right) \quad \text { as } h \rightarrow 0 .
$$

Thus, in case $\gamma_{0}$ and $\delta_{0}$ are real and $\gamma_{0}=\delta_{0}$, hence $\beta_{0}=\gamma_{0}=\delta_{0}$, there holds

$$
\widehat{Q}_{n}[f]-I[f]=O\left(h^{(\omega+1)(m+1)}\right) \quad \text { as } h \rightarrow 0 ; \quad \omega=\min \left\{\Re \gamma_{1}, \Re \delta_{1}\right\} .
$$

Proof. It is clear from Theorem 1.2 that we need to find the asymptotic expansions of the transformed integrand $\widehat{f}(t)=f(\psi(t)) \psi^{\prime}(t)$ as $t \rightarrow 0$ and $t \rightarrow 1$. Because $\psi(t) \rightarrow 0$ as $t \rightarrow 0$ and $\psi(t) \rightarrow 1$ as $t \rightarrow 1$, and recalling that $1-\psi(t)=\psi(1-t)$, we can obtain these by re-expanding the asymptotic series

$\widehat{f}(t) \sim \sum_{s=0}^{\infty} c_{s}[\psi(t)]^{\gamma_{s}} \psi^{\prime}(t) \quad$ as $\quad t \rightarrow 0, \quad \widehat{f}(t) \sim \sum_{s=0}^{\infty} d_{s}[\psi(1-t)]^{\delta_{s}} \psi^{\prime}(1-t) \quad$ as $\quad t \rightarrow 1$.

The $s$ th term in the first of these series contributes the sum

$$
K_{s}(t):=\sum_{i=0}^{\infty} e_{s i}^{(0)} t^{\gamma_{s}(m+1)+m+2 i} \quad \text { as } t \rightarrow 0, \quad e_{s 0}^{(0)}=c_{s} \epsilon_{0}^{\gamma_{s}+1} /(m+1) \neq 0,
$$

whereas the $s$ th term in the second series contributes the sum

$$
L_{s}(t):=\sum_{i=0}^{\infty} e_{s i}^{(1)}(1-t)^{\delta_{s}(m+1)+m+2 i} \quad \text { as } t \rightarrow 1, \quad e_{s 0}^{(1)}=d_{s} \epsilon_{0}^{\delta_{s}+1} /(m+1) \neq 0 .
$$

Thus, by Theorem 1.2, the most dominant terms in the expansion of $\widehat{Q}_{n}[f]-I[f]$ as $h \rightarrow 0$ are $e_{00}^{(0)} h^{\left(\gamma_{0}+1\right)(m+1)}$ and $e_{00}^{(1)} h^{\left(\delta_{0}+1\right)(m+1)}$. This proves part (i) of the theorem.

To prove part (ii), we recall from Theorem 1.2 and the remark following its proof that if we choose $m$ such that $\gamma_{s}(m+1)+m$ is an even integer, then all the powers of $t$ in the asymptotic expansion $K_{s}(t)$ of (4.1) are also even, hence do not contribute to the asymptotic expansion of $\widehat{Q}_{n}[f]-I[f]$. Similarly, if we choose $m$ such that $\delta_{s}(m+1)+m$ is an even integer, then all the powers of $(1-t)$ in the asymptotic expansion $L_{s}(t)$ of (4.2) are also even, hence do not contribute to the asymptotic expansion of $\widehat{Q}_{n}[f]-I[f]$. Now, we have either $\beta_{0}=\gamma_{0}$ or $\beta_{0}=\delta_{0}$. Thus, when $\beta_{0}$ is real, if we choose $m$ such that $\beta_{0}(m+1)+m=q$ is an even integer, then either $K_{0}(t)$ in (4.1) or $L_{0}(t)$ in (4.2) does not contribute to the asymptotic expansion 
of $\widehat{Q}_{n}[f]-I[f]$. This proves the first of the results in part (ii). The second result in part (ii) follows from this in a straightforward manner. This completes the proof.

Remark. Note that the results in part (ii) of Theorem 4.1 are made possible by our definition of the class $\mathcal{S}_{m}$ transformations, where we have excluded the powers $t^{m+1}, t^{m+3}, \ldots$, and $(1-t)^{m+1},(1-t)^{m+3}, \ldots$, from the asymptotic expansions of $\psi^{\prime}(t)$ as $t \rightarrow 0$ and $t \rightarrow 1$.

The situations described in the corollary below arise, for example, in case $f(x)$ is infinitely differentiable on $[0,1]$. The quality of $\widehat{Q}_{n}[f]$ in this corollary is best possible.

Corollary 4.2. Assume $f(x)$ is as in Theorem 4.1, and let the $\beta_{i}$ be as in part (ii) there. Then:

(i) In case $\beta_{0}=0$ and $\beta_{1}=1$, if we choose $m$ to be an even integer, we have $\widehat{Q}_{n}[f]-I[f]=O\left(h^{2 m+2}\right)$ as $h \rightarrow 0$.

(ii) In case $\beta_{0}=1$ and $\beta_{1}=2$, if we choose $m$ such that $2 m$ is an odd integer, we have $\widehat{Q}_{n}[f]-I[f]=O\left(h^{3 m+3}\right)$ as $h \rightarrow 0$.

(iii) In case $\beta_{0}=1$ and $\beta_{1}=3$, if we choose $m$ such that $2 m$ is an odd integer, we have $\widehat{Q}_{n}[f]-I[f]=O\left(h^{4 m+4}\right)$ as $h \rightarrow 0$.

Note that part (i) of the corollary applies when $|f(0)|+|f(1)| \neq 0$ and $\left|f^{\prime}(0)\right|+$ $\left|f^{\prime}(1)\right| \neq 0$. Part (ii) applies when $f(0)=f(1)=0,\left|f^{\prime}(0)\right|+\left|f^{\prime}(1)\right| \neq 0$, and $\left|f^{\prime \prime}(0)\right|+\left|f^{\prime \prime}(1)\right| \neq 0$. Part (iii) applies when $f(0)=f(1)=0,\left|f^{\prime}(0)\right|+\left|f^{\prime}(1)\right| \neq 0$, $f^{\prime \prime}(0)=f^{\prime \prime}(1)=0$, and $\left|f^{\prime \prime \prime}(0)\right|+\left|f^{\prime \prime \prime}(1)\right| \neq 0$.

Thus, the result of part (i) of Corollary 4.2, despite being quite good, is nevertheless inferior to those of parts (ii) and (iii). That is, the best accuracy that can be achieved by $\widehat{Q}_{n}[f]$ when $|f(0)|+|f(1)| \neq 0$ and $\left|f^{\prime}(0)\right|+\left|f^{\prime}(1)\right| \neq 0$ is less than those achieved when $f(0)=f(1)=0$. In the next theorem, we show how this situation can be improved in a simple way.

Theorem 4.3. Assume $f \in C^{\infty}[0,1]$ and that $|f(0)|+|f(1)| \neq 0$; that is, at least one of $f(0)$ and $f(1)$ is nonzero. Let $p(x)$ be the linear function that interpolates $f(x)$ at $x=0$ and $x=1$, and let $u(x)=f(x)-p(x)$. Next, transform the variable $x$ in the integral $\int_{0}^{1} u(x) d x$ via $x=\psi(t)$, where $\psi \in \mathcal{S}_{m}$, and approximate the transformed integral by the trapezoidal rule. Denote the resulting approximations by $\widehat{Q}_{n}[u]$. Then

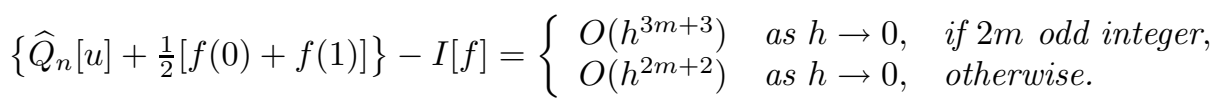

Proof. First,

$$
\int_{0}^{1} f(x) d x=\int_{0}^{1} u(x) d x+\int_{0}^{1} p(x) d x=\int_{0}^{1} u(x) d x+\frac{1}{2}[f(0)+f(1)] .
$$

Next, we note that $u \in C^{\infty}[0,1]$ and vanishes at $x=0$ and $x=1$. The result now follows by applying part (i) of Theorem 4.1 and part (ii) of Corollary 4.2 to the integral $\int_{0}^{1} u(x) d x$.

In case only one of $f(0)$ and $f(1)$ vanishes, and a few other special conditions hold, we can use another approach, quite different from that described in Theorem 
4.3. We give this approach next. Note the rather unusual application of the class $\mathcal{S}_{m}$ transformations.

Theorem 4.4. Assume $f \in C^{\infty}[0,1]$ and that only one of $f(0)$ and $f(1)$ vanishes. Let $f(0)=0$, without loss of generality, and assume that $f^{(i)}(0)=0$ for $2 \leq$ $i \leq j-1$, and $f^{(j)}(0) \neq 0$ for some $j \geq 2$. Assume also that $f^{(2 k+1)}(1)=0$, $k=0,1, \ldots$. Let $\psi(t)$ be in $\mathcal{S}_{m}$ for some $m$, and transform the variable $x$ via $x=\bar{\psi}(t)=2 \psi(t / 2)$. Thus,

$$
I[f]=\int_{0}^{1} f(x) d x=\int_{0}^{1} \bar{f}(t) d t ; \quad \bar{f}(t)=f(\bar{\psi}(t)) \bar{\psi}^{\prime}(t)=f(2 \psi(t / 2)) \psi^{\prime}(t / 2) .
$$

Let

$$
\bar{Q}_{n}[f]=h\left[\sum_{i=1}^{n-1} \bar{f}(i h)+\frac{1}{2} \bar{f}(1)\right], \quad h=\frac{1}{n} .
$$

Then, whether $f^{\prime}(0) \neq 0$ or not,

$$
\bar{Q}_{n}[f]-I[f]= \begin{cases}O\left(h^{(j+1)(m+1)}\right) \quad \text { as } h \rightarrow 0, & \text { if } 2 m \text { odd integer }, \\ O\left(h^{2 m+2}\right) \text { as } h \rightarrow 0, & \text { otherwise. }\end{cases}
$$

Thus, when $2 m$ is an odd integer, $\bar{Q}_{n}[f]-I[f]=O\left(h^{3 m+3}\right)$ as $h \rightarrow 0$, at worst. In case $f^{\prime}(0)=0$, the result above can be refined as follows:

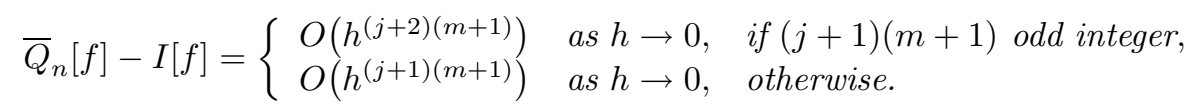

Thus, in case $f^{\prime}(0)=0$, when $(j+1)(m+1)$ is an odd integer, $\bar{Q}_{n}[f]-I[f]=$ $O\left(h^{4 m+4}\right)$ as $h \rightarrow 0$, at worst.

Proof. We first analyze the behavior of $\bar{\psi}(t)$ as $t \rightarrow 0$ and as $t \rightarrow 1$. Clearly, $\bar{\psi}(0)=0, \bar{\psi}(1)=1$, and $\bar{\psi}(t)$ is increasing on $[0,1]$. From Definition 2.1.

$$
\bar{\psi}^{\prime}(t) \sim \sum_{i=0}^{\infty} \bar{\epsilon}_{i}^{(0)} t^{m+2 i} \quad \text { as } t \rightarrow 0,
$$

hence

$$
\bar{\psi}_{i}(t) \sim \sum_{i=0}^{\infty} \frac{\bar{\epsilon}_{i}^{(0)}}{m+2 i+1} t^{m+2 i+1} \quad \text { as } t \rightarrow 0 .
$$

From the fact that $\psi^{\prime}(1-t)=\psi^{\prime}(t)$, we have $\psi^{(2 k)}(1 / 2)=0, k=1,2, \ldots$. Consequently,

$$
\bar{\psi}^{\prime}(t) \sim \sum_{i=0}^{\infty} \bar{\epsilon}_{i}^{(1)}(1-t)^{2 i} \quad \text { as } t \rightarrow 1
$$

hence

$$
\bar{\psi}(t) \sim 1-\sum_{i=0}^{\infty} \frac{\bar{\epsilon}_{i}^{(1)}}{2 i+1}(1-t)^{2 i+1} \quad \text { as } t \rightarrow 1 .
$$

By the conditions imposed on the function $f(x)$, we have

$$
f(x) \sim c_{1} x+\sum_{s=j}^{\infty} c_{s} x^{s} \quad \text { as } x \rightarrow 0, \quad c_{1} \neq 0 \text { in general }
$$


and

$$
f(x) \sim \sum_{s=0}^{\infty} d_{s}(1-x)^{2 s} \quad \text { as } x \rightarrow 1 .
$$

Let us now substitute the asymptotic expansions of $\bar{\psi}(t)$ and $\bar{\psi}^{\prime}(t)$ in the expansions

$$
\bar{f}(t) \sim c_{1} \bar{\psi}(t) \bar{\psi}^{\prime}(t)+\sum_{s=j}^{\infty} c_{s}[\bar{\psi}(t)]^{s} \bar{\psi}^{\prime}(t) \quad \text { as } t \rightarrow 0
$$

and

$$
\bar{f}(t) \sim \sum_{s=0}^{\infty} d_{s}[1-\bar{\psi}(t)]^{2 s} \bar{\psi}^{\prime}(t) \quad \text { as } t \rightarrow 1
$$

We obtain

$$
\bar{f}(t) \sim \sum_{i=0}^{\infty} e_{1 i}^{(0)} t^{2 m+1+2 i}+\sum_{s=j}^{\infty} \sum_{i=0}^{\infty} e_{s i}^{(0)} t^{s(m+1)+m+2 i} \quad \text { as } t \rightarrow 0
$$

and

$$
\bar{f}(t) \sim \sum_{i=0}^{\infty} e_{i}^{(1)}(1-t)^{2 i} \quad \text { as } t \rightarrow 1 .
$$

Proceeding as in the proof of Theorem 4.1, we complete the proof. We leave the details to the reader.

Note that, under the transformation $\bar{\psi}(t)$, the transformed abscissas $x_{i}=\bar{\psi}(i / n)$ are clustered in a small right neighborhood of $x=0$; no clustering takes place near $x=1$.

In connection with the use of $\bar{\psi}(t)=2 \psi(t / 2)$ as the variable transformation in Theorem 4.4 it is interesting to note that, under the condition that $f^{(2 k+1)}(1)=0$ for all $k \geq 0$, there is no contribution to the expansion of $\bar{Q}_{n}[f]-I[f]$ as $h \rightarrow 0$ from the endpoint $x=1$. This is so for all values of $m$.

In case $f(0) \neq 0$ and $f(1)=0$ in Theorem 4.4 we write $I[f]=\int_{0}^{1} g(x) d x$, with $g(x)=f(1-x)$, and apply the method described there with $f(x)$ replaced by $g(x)$.

Finally, we note that the use of $\bar{\psi}(t)=2 \psi(t / 2)$, with any variable transformation $\psi(t) \in C^{\infty}[0,1]$ for which $\psi(0)=0, \psi(1)=1, \psi^{\prime}(t)>0$ on $(0,1)$, and $\psi^{\prime}(t)$ is symmetric with respect to $t=1 / 2$, that is, $\psi^{\prime}(1-t)=\psi^{\prime}(t)$ hence $\psi(1-t)=1-\psi(t)$, was introduced by Johnston 8 in conjunction with Gauss-Legendre quadrature in the context of computing $\int_{0}^{1} f(x) d x$ with a singularity at $x=0$ only. Of course, our motivation and usage of $\bar{\psi}(t)$ (only with $\psi \in \mathcal{S}_{m}$ and with special values of $m$ ) in the present work are completely different from those of $[8]$.

Remark. The situations described in part (iii) of Corollary 4.2 and in Theorems 4.3 and 4.4 arise naturally in integration of functions over an arbitrary smooth surface $S$ in $\mathbb{R}^{3}$, after this surface has been mapped to the surface of the unit sphere, and after the transformed integral has been expressed in terms of surface spherical coordinates $\theta$ and $\phi(x=\sin \theta \cos \phi, y=\sin \theta \sin \phi, z=\cos \theta$, as usual), the "effective" integral being of the form $\int_{0}^{\pi} v(\theta) d \theta$ :

1. Part (iii) of Corollary 4.2 applies to the case in which the integrand functions are smooth over the surface $S$. In this case, it turns out that $v(\theta) \sim$ $\sum_{i=0}^{\infty} a_{i} \theta^{2 i+1}$ as $\theta \rightarrow 0$ and $v(\theta) \sim \sum_{i=0}^{\infty} b_{i}(\pi-\theta)^{2 i+1}$ as $\theta \rightarrow \pi$. Thus, 
the error in the approximation to $\int_{0}^{\pi} v(\theta) d \theta$ is at worst $O\left(h^{4 m+4}\right)$ when $\psi \in \mathcal{S}_{m}$ with $2 m$ an odd integer.

2. Theorems 4.3 and 4.4 apply to the case in which the integrand functions are smooth over the surface $S$, except for a point singularity of the singleor double-layer type. In particular, for integration over the surface of the unit sphere, and with the point singularity at the south pole $\theta=\pi$, there holds $v(\theta) \sim \sum_{i=0}^{\infty} a_{i} \theta^{2 i+1}$ as $\theta \rightarrow 0$ and $v(\theta) \sim \sum_{i=0}^{\infty} b_{i}(\pi-\theta)^{2 i}$ as $\theta \rightarrow \pi$. Thus, the conditions of Theorem 4.4 are satisfied by $v(\theta)$ with $j \geq 3$, and the error in the approximation to $\int_{0}^{\pi} v(\theta) d \theta$ is at worst $O\left(h^{4 m+4}\right)$ when $\psi \in \mathcal{S}_{m}$ with $2 m$ an odd integer.

For these problems and their treatment via the extended class $\mathcal{S}_{m}$ variable transformations, we refer the reader to the report [18] and the recent papers 21] and 22 by Sidi. Actually, it was these problems that motivated the extension of the class $\mathcal{S}_{m}$.

\section{Numerical EXAMPLES}

In this section, we provide two examples to illustrate the validity of the results of Section 4 numerically. The computations for these examples were done in quadruple-precision arithmetic (approximately 35 decimal digits).

Example 5.1. Let us consider the integral

$$
\int_{0}^{1} \frac{x(1-x)}{1+x} d x=\frac{3}{2}-2 \log 2 .
$$

We use the trapezoidal rule after transforming the integral with the $\sin ^{m}$-transformation for various values of $m$. Note that the integrand is as in part (ii) of Corollary 4.2. The numerical results in Tables 1 and 2 illustrate the results of Theorem 4.1 and Corollary 4.2

Table 1 gives the relative errors in the $\widehat{Q}_{n}[f], n=2^{k}, k=1,2, \ldots, 10$, for $m=j / 2, j=3,4, \ldots, 12$. Table 2 presents the numbers

$$
\mu_{m, k}=\frac{1}{\log 2} \cdot \log \left(\frac{\left|\widehat{Q}_{2^{k}}[f]-I[f]\right|}{\left|\widehat{Q}_{2^{k+1}}[f]-I[f]\right|}\right)
$$

for the same values of $m$ and for $k=1,2, \ldots, 9$. It is seen that, with increasing $k$, the $\mu_{m, k}$ are tending to $2 m+2$ when $m$ is an integer, in accordance with part (i) of Theorem 4.1, and to $3 m+3$ when $2 m$ is an odd integer, in accordance with part (ii) of Theorem 4.1 and part (ii) of Corollary 4.2 ,

Example 5.2. Consider the integral

$$
\int_{0}^{1} \frac{\sin \left(\frac{1}{2} \pi x\right)}{1+(1-x)^{2}} d x=0.549122163208195 \cdots .
$$

It is not difficult to see that the integrand satisfies the conditions of Theorem 4.4 with $j=2$ there. Therefore, we transform the variable $x$ via $x=\bar{\psi}(t)$, where $\bar{\psi}(t)=2 \psi(t / 2), \psi(t)$ being in $\mathcal{S}_{m}$ for some $m$, and apply the trapezoidal rule $\bar{Q}_{n}[f]$ described in Theorem 4.4. Thus, Theorem 4.4 applies with $j=2$.

The numerical results in Tables 3 and 4 illustrate the results of Theorem 4.4 . 
TABLE 1. Relative errors in the rules $\widehat{Q}_{n}[f]$ for the integral of Example 5.1 in Section 5 , obtained with $n=2^{k}$,

$k=1(1) 10$, and with the $\sin ^{m}$-transformation using $m=1.5(0.5) 6$.

\begin{tabular}{||r||c|c|c|c|c|c|c|c|c|c|}
\hline$n$ & $m=1.5$ & $m=2.0$ & $m=2.5$ & $m=3.0$ & $m=3.5$ & $m=4.0$ & $m=4.5$ & $m=5.0$ & $m=5.5$ & $m=6.0$ \\
\hline \hline 2 & $3.17 D-01$ & $4.66 D-01$ & $6.01 D-01$ & $7.27 D-01$ & $8.44 D-01$ & $9.54 D-01$ & $1.06 D+00$ & $1.16 D+00$ & $1.25 D+00$ & $1.35 D+00$ \\
4 & $6.30 D-04$ & $5.53 D-03$ & $2.39 D-03$ & $9.66 D-03$ & $2.96 D-02$ & $5.57 D-02$ & $8.65 D-02$ & $1.20 D-01$ & $1.57 D-01$ & $1.94 D-01$ \\
8 & $1.79 D-06$ & $2.83 D-05$ & $5.20 D-08$ & $6.61 D-06$ & $5.69 D-06$ & $3.08 D-06$ & $1.55 D-05$ & $6.71 D-05$ & $1.56 D-04$ & $2.69 D-04$ \\
16 & $8.47 D-09$ & $4.14 D-07$ & $1.52 D-10$ & $1.81 D-08$ & $7.48 D-12$ & $1.39 D-09$ & $4.08 D-12$ & $1.99 D-10$ & $2.97 D-11$ & $2.18 D-10$ \\
32 & $4.53 D-11$ & $6.37 D-09$ & $9.71 D-14$ & $6.77 D-11$ & $5.37 D-16$ & $1.24 D-12$ & $6.19 D-18$ & $3.46 D-14$ & $1.31 D-19$ & $1.38 D-15$ \\
64 & $2.48 D-13$ & $9.91 D-11$ & $6.57 D-17$ & $2.62 D-13$ & $4.41 D-20$ & $1.18 D-15$ & $6.04 D-23$ & $8.09 D-18$ & $1.48 D-25$ & $7.86 D-20$ \\
128 & $1.37 D-15$ & $1.55 D-12$ & $4.51 D-20$ & $1.02 D-15$ & $3.76 D-24$ & $1.14 D-18$ & $6.36 D-28$ & $1.95 D-21$ & $1.91 D-31$ & $4.72 D-24$ \\
256 & $7.56 D-18$ & $2.42 D-14$ & $3.11 D-23$ & $3.98 D-18$ & $3.23 D-28$ & $1.12 D-21$ & $7.22 D-33$ & $4.76 D-25$ & $3.85 D-34$ & $2.87 D-28$ \\
512 & $4.18 D-20$ & $3.77 D-16$ & $2.15 D-26$ & $1.55 D-20$ & $2.73 D-32$ & $1.09 D-24$ & $7.70 D-34$ & $1.16 D-28$ & $1.93 D-34$ & $1.70 D-32$ \\
1024 & $2.31 D-22$ & $5.90 D-18$ & $1.48 D-29$ & $6.07 D-23$ & $6.74 D-34$ & $1.06 D-27$ & $7.70 D-34$ & $2.93 D-32$ & $7.70 D-34$ & $3.85 D-34$ \\
\hline
\end{tabular}

TABLE 2. The numbers $\mu_{m, k}=\frac{1}{\log 2} \cdot \log \left(\frac{\left|\widehat{Q}_{2^{k}}[f]-I[f]\right|}{\left|\widehat{Q}_{2^{k+1}}[f]-I[f]\right|}\right)$, for $k=1(1) 9$ and $m=1.5(0.5) 6$, for the integral of Example 5.1 in Section $\left[5\right.$, where $\widehat{Q}_{n}[f]$ are those of Table 1 .

\begin{tabular}{||c||c|c|c|c|c|c|c|c|c|c||}
\hline$k$ & $m=1.5$ & $m=2.0$ & $m=2.5$ & $m=3.0$ & $m=3.5$ & $m=4.0$ & $m=4.5$ & $m=5.0$ & $m=5.5$ & $m=6.0$ \\
\hline \hline 1 & 8.976 & 6.397 & 7.977 & 6.234 & 4.835 & 4.099 & 3.614 & 3.265 & 3.001 & 2.794 \\
2 & 8.459 & 7.609 & 15.486 & 10.513 & 12.342 & 14.142 & 12.444 & 10.810 & 9.968 & 9.492 \\
3 & 7.724 & 6.095 & 8.416 & 8.514 & 19.538 & 11.112 & 21.859 & 18.367 & 22.329 & 20.239 \\
4 & 7.547 & 6.024 & 10.613 & 8.061 & 13.765 & 10.137 & 19.331 & 12.488 & 27.758 & 17.266 \\
5 & 7.511 & 6.006 & 10.530 & 8.016 & 13.572 & 10.033 & 16.644 & 12.061 & 19.752 & 14.100 \\
6 & 7.503 & 6.001 & 10.507 & 8.004 & 13.518 & 10.008 & 16.535 & 12.015 & 19.563 & 14.025 \\
7 & 7.501 & 6.000 & 10.502 & 8.001 & 13.505 & 10.002 & 16.426 & 12.004 & 8.954 & 14.006 \\
8 & 7.500 & 6.000 & 10.500 & 8.000 & 13.529 & 10.001 & $*$ & 12.001 & $*$ & 14.038 \\
9 & 7.500 & 6.000 & 10.500 & 8.000 & $*$ & 10.000 & $*$ & 11.954 & $*$ & $*$ \\
\hline
\end{tabular}


TABLE 3. Relative errors in the rules $\bar{Q}_{n}[f]$ for the integral of Example 5.2 in Section 5 , obtained with $n=2^{k}$, $k=1(1) 9$, and with the $\sin ^{m}$-transformation using $m=1.5(0.5) 6$.

\begin{tabular}{||r||c|c|c|c|c|c|c|c|c|c||}
\hline$n$ & $m=1.5$ & $m=2.0$ & $m=2.5$ & $m=3.0$ & $m=3.5$ & $m=4.0$ & $m=4.5$ & $m=5.0$ & $m=5.5$ & $m=6.0$ \\
\hline \hline 2 & $3.37 D-02$ & $6.41 D-02$ & $1.04 D-01$ & $1.50 D-01$ & $2.00 D-01$ & $2.53 D-01$ & $3.06 D-01$ & $3.60 D-01$ & $4.14 D-01$ & $4.67 D-01$ \\
4 & $7.93 D-05$ & $2.86 D-04$ & $7.94 D-04$ & $1.69 D-03$ & $3.09 D-03$ & $5.08 D-03$ & $7.73 D-03$ & $1.11 D-02$ & $1.51 D-02$ & $1.98 D-02$ \\
8 & $5.27 D-09$ & $1.73 D-07$ & $4.39 D-08$ & $2.07 D-07$ & $6.66 D-07$ & $1.80 D-06$ & $4.18 D-06$ & $8.58 D-06$ & $1.60 D-05$ & $2.78 D-05$ \\
16 & $2.61 D-11$ & $2.76 D-09$ & $5.61 D-14$ & $2.94 D-11$ & $3.06 D-14$ & $3.09 D-13$ & $1.22 D-12$ & $5.14 D-12$ & $1.79 D-11$ & $5.39 D-11$ \\
32 & $1.44 D-13$ & $4.30 D-11$ & $3.80 D-17$ & $1.13 D-13$ & $2.55 D-20$ & $5.11 D-16$ & $3.50 D-23$ & $3.51 D-18$ & $2.24 D-23$ & $3.39 D-20$ \\
64 & $7.92 D-16$ & $6.71 D-13$ & $2.61 D-20$ & $4.42 D-16$ & $2.17 D-24$ & $4.96 D-19$ & $3.68 D-28$ & $8.48 D-22$ & $1.11 D-31$ & $2.05 D-24$ \\
128 & $4.37 D-18$ & $1.05 D-14$ & $1.80 D-23$ & $1.72 D-18$ & $1.87 D-28$ & $4.84 D-22$ & $2.89 D-33$ & $2.06 D-25$ & $1.93 D-34$ & $1.24 D-28$ \\
256 & $2.41 D-20$ & $1.64 D-16$ & $1.24 D-26$ & $6.74 D-21$ & $1.73 D-32$ & $4.73 D-25$ & $8.67 D-34$ & $5.04 D-29$ & $3.85 D-34$ & $7.70 D-33$ \\
512 & $1.33 D-22$ & $2.56 D-18$ & $8.58 D-30$ & $2.63 D-23$ & $1.06 D-33$ & $4.61 D-28$ & $1.25 D-33$ & $1.25 D-32$ & $1.93 D-34$ & $0.00 D+00$ \\
\hline
\end{tabular}

TABLE 4. The numbers $\mu_{m, k}=\frac{1}{\log 2} \cdot \log \left(\frac{\left|\bar{Q}_{2 k}[f]-I[f]\right|}{\left|\bar{Q}_{2^{k+1}}[f]-I[f]\right|}\right)$, for $k=1(1) 7$ and $m=1.5(0.5) 6$, for the integral of Example $\left[5.2\right.$ in Section $\left[5\right.$, where $\bar{Q}_{n}[f]$ are those of Table 3 .

\begin{tabular}{||c||c|c|c|c|c|c|c|c|c|c||}
\hline$k$ & $m=1.5$ & $m=2.0$ & $m=2.5$ & $m=3.0$ & $m=3.5$ & $m=4.0$ & $m=4.5$ & $m=5.0$ & $m=5.5$ & $m=6.0$ \\
\hline \hline 1 & 8.730 & 7.806 & 7.030 & 6.469 & 6.017 & 5.636 & 5.309 & 5.025 & 4.776 & 4.556 \\
2 & 13.878 & 10.690 & 14.144 & 12.999 & 12.180 & 11.460 & 10.853 & 10.333 & 9.879 & 9.479 \\
3 & 7.655 & 5.973 & 19.578 & 12.782 & 24.375 & 22.479 & 21.712 & 20.670 & 19.772 & 18.978 \\
4 & 7.508 & 6.006 & 10.527 & 8.016 & 20.197 & 9.238 & 35.015 & 20.483 & 39.538 & 30.566 \\
5 & 7.502 & 6.001 & 10.508 & 8.004 & 13.518 & 10.008 & 16.540 & 12.015 & 27.595 & 14.016 \\
6 & 7.501 & 6.000 & 10.502 & 8.001 & 13.504 & 10.002 & 16.957 & 12.004 & 9.167 & 14.006 \\
7 & 7.500 & 6.000 & 10.500 & 8.000 & 13.397 & 10.001 & 1.737 & 12.001 & -1.000 & 13.978 \\
\hline
\end{tabular}


Table 3 gives the relative errors in $\bar{Q}_{n}[f], n=2^{k}, k=1,2, \ldots, 9$, for $m=j / 2$, $j=3,4, \ldots, 12$. Table 4 presents the numbers

$$
\mu_{m, k}=\frac{1}{\log 2} \cdot \log \left(\frac{\left|\bar{Q}_{2^{k}}[f]-I[f]\right|}{\left|\bar{Q}_{2^{k+1}}[f]-I[f]\right|}\right)
$$

for the same values of $m$ and for $k=1,2, \ldots, 8$. It is seen that, with increasing $k$, the $\mu_{m, k}$ are tending to $2 m+2$ when $m$ is an integer, and to $3 m+3$ when $2 m$ is an odd integer, in accordance with Theorem 4.4

Let us denote by $\check{Q}_{n, m}[f]$ either of the transformed trapezoidal rules $\widehat{Q}_{n}[f]$ or $\bar{Q}_{n}[f]$ resulting from a variable transformation $\psi \in \mathcal{S}_{m}$. From Tables 2 and 4 , we see that the accuracy of $\check{Q}_{n, m}[f]$, for fixed $n$, first increases with increasing $m$, reaches a peak, and then starts to decrease. In other words, for a given $n$, there seems to be a "best" value of $m$. We also see that this value of $m$ increases as $n$ increases. An interesting question now is whether we can quantify this phenomenon. Formally, for a class $U$ of functions $f(x), I[f] \neq 0$, we would like to know $\widetilde{m}=\widetilde{m}(n)$, for which

$$
E_{n, \widetilde{m}}=\max _{f \in U} \min _{m} E_{n, m}, \quad E_{n, m}=\left|\frac{\check{Q}_{n, m}[f]-I[f]}{I[f]}\right| .
$$

Of course, this is a difficult question to answer theoretically. By looking at Tables 2 and 4 , however, we could perhaps say the following about how $\widetilde{m}$ increases as a function of $n$ for the classes of functions in Examples 5.1 and 5.2. If for a particular $n, E_{n, m^{\prime}}$ is the smallest of the $E_{n, m}$, then $E_{2 n, m^{\prime}+1}$ seems to be the smallest of the $E_{2 n, m}$. A general rule of thumb concerning the best value of $m$ for all classes of functions seems to be difficult to reach at this stage, however.

\section{ACKNOWLEDGMENTS}

The author would like to thank the two anonymous referees for their critical comments and illuminating discussions that have helped to improve the contents and style of this work.

\section{REFERENCES}

[1] M. Abramowitz and I.A. Stegun. Handbook of Mathematical Functions with Formulas, Graphs, and Mathematical Tables. Number 55 in Nat. Bur. Standards Appl. Math. Series. U.S. Government Printing Office, Washington, D.C., 1964. MR0167642 (29:4914)

[2] K.E. Atkinson. An Introduction to Numerical Analysis. Wiley, New York, second edition, 1989. MR1007135 (90m:65001)

[3] P.J. Davis and P. Rabinowitz. Methods of Numerical Integration. Academic Press, New York, second edition, 1984. MR0760629 (86d:65004)

[4] D. Elliott. Sigmoidal transformations and the trapezoidal rule. J. Austral. Math. Soc., Series B (E), 40:E77-E137, 1998. MR1658346 (99j:65030)

[5] W. Gautschi. Algorithm 726: ORTHPOL - a package of routines for generating orthogonal polynomials and Gauss-type quadrature rules. ACM Transactions on Mathematical Software, 20:21-62, 1994 .

[6] M. Hill and I. Robinson. d2lri: A nonadaptive algorithm for two-dimensional cubature. J. Comp. Appl. Math., 112:121-145, 1999. MR.1728455

[7] M. Iri, S. Moriguti, and Y. Takasawa. On a certain quadrature formula. Kokyuroku of Res. Inst. for Math. Sci. Kyoto Univ., 91:82-118, 1970. In Japanese. English translation in J. Comp. Appl. Math., 17:3-20, 1987. MR0884257(88j:65057)

[8] P.R. Johnston. Semi-sigmoidal transformations for evaluating weakly singular boundary element integrals. Intern. J. Numer. Methods Engrg., 47:1709-1730, 2000. MR1750249 
[9] N.M. Korobov. Number-Theoretic Methods of Approximate Analysis. GIFL, Moscow, 1963. In Russian. MR0157483 (28:716)

[10] D.P. Laurie. Periodizing transformations for numerical integration. J. Comp. Appl. Math., 66:337-344, 1996. MR 1393741

[11] D. Levin. Development of non-linear transformations for improving convergence of sequences. Intern. J. Computer Math., B3:371-388, 1973. MR0359261(50:11716)

[12] M. Mori. An IMT-type double exponential formula for numerical integration. Publ. Res. Inst. Math. Sci. Kyoto Univ., 14:713-729, 1978. MR0527197 (81c:65012)

[13] A. Ralston and P. Rabinowitz. A First Course in Numerical Analysis. McGraw-Hill, New York, second edition, 1978. MR0494814 (58:13599)

[14] I. Robinson and M. Hill. Algorithm 816: r2d2lri: An algorithm for automatic two-dimensional cubature. ACM Trans. Math. Software, 28:75-100, 2002.

[15] T.W. Sag and G. Szekeres. Numerical evaluation of high-dimensional integrals. Math. Comp., 18:245-253, 1964. MR0165689 (29:2969)

[16] D. Shanks. Nonlinear transformations of divergent and slowly convergent sequences. J. Math. and Phys., 34:1-42, 1955. MR0068901 (16:961e)

[17] A. Sidi. A new variable transformation for numerical integration. In H. Brass and G. Hämmerlin, editors, Numerical Integration IV, number 112 in ISNM, pages 359-373, Birkhäuser, Basel, 1993. MR1248416 (94k:65032)

[18] A. Sidi. Class $\mathcal{S}_{m}$ variable transformations and application to numerical integration over smooth surfaces in $\mathbb{R}^{3}$. Preprint. Computer Science Dept., Technion-Israel Institute of Technology, 2003.

[19] A. Sidi. Practical Extrapolation Methods: Theory and Applications. Number 10 in Cambridge Monographs on Applied and Computational Mathematics. Cambridge University Press, Cambridge, 2003. MR 1994507(2004e:65005)

[20] A. Sidi. Euler-Maclaurin expansions for integrals with endpoint singularities: a new perspective. Numer. Math., 98:371-387, 2004. MR2092747

[21] A. Sidi, Numerical integration over smooth surfaces in $\mathbb{R}^{3}$ via class $\mathcal{S}_{m}$ variable transformations. Part I: Smooth integrands. Appl. Math. Comp., 2005. In press. First published electronically on April 11, 2005.

[22] A. Sidi, Application of class $\mathcal{S}_{m}$ variable transformations to numerical integration over surfaces of spheres. J. Comp. Appl. Math., 2005. In press. First published electronically on April $11,2005$.

[23] I.H. Sloan and S. Joe. Lattice Methods in Multiple Integration. Clarendon Press, Oxford, 1994. MR1442955 (98a:65026)

[24] P. Verlinden, D.M. Potts, and J.N. Lyness. Error expansions for multidimensional trapezoidal rules with Sidi transformations. Numer. Algorithms, 16:321-347, 1997. MR 1617168 (99h:65039)

[25] P. Wynn. On a device for computing the $e_{m}\left(S_{n}\right)$ transformation. Mathematical Tables and Other Aids to Computation, 10:91-96, 1956. MR0084056 (18:801e)

Computer Science Department, Technion - Israel Institute of Technology, Haifa 32000 , ISRAEL

E-mail address: asidi@cs.technion.ac.il

$U R L:$ http://www.cs.technion.ac.il/ asidi/ 